\title{
Hydrogen Bonding - The Key to Desalination (A Review)
}

\author{
Dr S. J. Purohit ${ }^{1}$, Rajan Mishra ${ }^{2}$, Akhil Subramanian ${ }^{3}$
}

${ }^{1}$ Dr S. J. Purohit (HOD, DEPT of Chemical Engineering- Thadomal Shahani Engineering College, Mumbai)

${ }^{2}$ Rajan Mishra (BE- Thadomal Shahani Engineering College, Mumbai)

${ }^{3}$ Akhil Subramanian (BE- Thadomal Shahani Engineering College, Mumbai)

\begin{abstract}
Energy crisis today is a major matter of concern. Energy is the most essential ingredient in the process of economic growth and development. The search for alternative sources for various unit operations such as evaporation, drying, distillation, etc. has been in continuum but without much success. Industries being the major consumers of energy, its efficient usage and minimal wastage are of profound importance.
\end{abstract}

ETPs or Effluent Treatment Plants are one of the components of a majority of the industry where a lot of energy is consumed. In every ETP, separate equipments are present to treat salt water. Desalination techniques such as Flash and Multi Effect Distillation in thermal, Electro dialysis and Reverse Osmosis $(R O)$ in membrane category and many other processes such as freezing and humidification are used. For instance RO today is the most commonly used method for desalination. But the problem that accompanies it is that RO rejects concentrated brine solution every time it is used. This massive tonnage of salt water is then sent through a set of Multi-Effect Evaporators which not only increases the cost but majorly increases the energy requirement. The real problem with all the methods used is the need optimum economic designs and higher efficiency requirements thus making it both eco-friendly and economical for the industry. Modifications and innovations such as Incorporation of vacuum in humidification system increases the evaporation rate of water due to reduction in boiling point. In Electrodialysis, ion exchange resins are incorporated between the electrodes which help separate out ions into continuous streams. These provide continuity to the process and also enhance its rate. But still the need for energy turns out to be the major issue. This paper suggests an alternative innovative technique reduction in $h$ bonding of water that can virtually eliminate the excessive energy needs in these processes.

Keywords-Desalination, RO, Electrodialysis, Humidification, Solvents.

\section{INTRODUCTION}

Desalination of water is a major concern for both industries as well as for municipal supply across the globe. The demand for freshwater is ever increasing .ie demand for freshwater in the MENA(Middle East and North Africa Region) is estimated to go from 42 today to $200 \mathrm{~km}^{3}$ in year 2050[1].Also industries that include Oil \& Gas, Refining \& Petrochemicals, Power Generation Food and Beverage, Pharmaceutical, Microelectronics, Pulp \& Paper, and Mining industries also make use of various desalination technologies to obtain freshwater for different purposes[2]. The crisis doesn't only include the costs of maintenance and running of desalination units but also the environmental impact that these methods are causing. For example Saudi Arabia alone is burning 1.5 million barrels of crude oil per day equivalent to produce freshwater through desalination releasing massive amounts of greenhouse gases into the atmosphere [1].

This paper is an attempt to describe the currently used methods and the problems associated with them both in operation as well as maintenance. Also an alternative innovative technologies that can be adapted instead of or in combination with the current technologies.

\section{EXISTING METHODS:}

Commercial technologies for desalination include membrane separation processes such as reverse osmosis (RO) and electro dialysis (ED), multi effect distillation (MED), multistage flash (MSF) and vapour compression distillation (VCD). These technologies are the most widely used desalination processes with MSF and RO with a total share of about 78\% [3].Also the use of direct solar energy for desalinating seawater is being investigated quite extensively.

The issues faced in the most common methods are listed as follows are listed as follows: 


\subsection{Membrane technology}

This mainly includes RO and ED. In RO high pressures up to 70-90 bars is required and for every single unit of freshwater produced around 1.5 times of that amount is recovered as reject[1].The common problem of fouling and scaling is also a major issue but can be controlled by pre-treatment can reduce these to a certain extent. Fouling of membranes is a major issue in spite of advances in membrane technology. Problems of concentration polarisation also persist. Adaptability i.e.: variation in feed concentration also causes trouble in setting of membrane based processes.

\subsection{Thermal Technologies}

These are the most commonly used especially in regions with high availability of energy sources. These include MSF, MED, VCD, etc. These are the most commonly used especially in regions with high availability of energy sources. These include MSF, MED, VCD, etc.

The main issues with these are fouling, high energy requirements, larger footprint requirement, corrosion problems and most of all environmental pollution due to extensive use of fossil fuels as energy resources. Use of vacuum for higher efficiency is also used but is a costly affair.

\subsection{Renewable Energy Powered Conventional Desalination}

Most promising energy source that is being investigated to replace various sources of energy is solar. These can be broadly classified into 2 categories concentrating solar power (CSP) technology and photovoltaic (PV) technology.

$\mathrm{PV}$ is conversion of solar radiations to electricity which in turn is used in various desalination processes. But the lower efficiency of PV panels, high setup costs and large area requirements limits its application in this particular field.

CSP technology is the most promising methodology to extract solar energy that would take the mantle as the most viable and eco-friendly alternative to current sources of energy in desalination. Various models have been proposed that include use of parabolic trough collectors, Fresnel lenses, solar towers, etc. but the current available technology is insufficient in meeting the world's requirements [7]

Also other sources of renewable energy such as wind, geothermal etc. have also been proposed but none of these sources with today's technology are potent enough to satisfy current energy needs. Thus more of R\&D and vigorous improvement in efficiencies might make these sources a viable solution in the future but as of now the issue of energy crisis persists.

\section{SUGGESTED SOLUTION}

The current area of research focuses on either optimising the current methods or finding greener and cheaper alternatives to meet the current and future's humongous energy needs. In this paper we propose an alternative means of desalination that minimises, rather virtually eliminates the need for high energy sources. The method is as follows:

\subsection{Reduction of H-bonding}

Hydrogen bonding forms in liquid water as the hydrogen atoms of one water molecule are attracted towards the oxygen atom of a neighboring water molecule; generally, a proton shared by two lone electron pairs. In a water molecule (H2O), the oxygen nucleus with +8 charges attracts electrons better than the hydrogen nucleus with its +1 charge. Hence, the oxygen atom is partially negatively charged and the hydrogen atom is partially positively charged. The hydrogen atoms are not only covalently attached to their oxygen atoms but also attracted towards other nearby oxygen atoms. This attraction is the basis of the 'hydrogen' bonds. Hydrogen bonds arise in water where each partially positively charged hydrogen atom is covalently attached to a partially negatively charged oxygen from a water molecule with bond energy of about $492 \mathrm{KJ} / \mathrm{mol}$ and is also attracted, but much more weakly, to a neighboring partially negatively charged oxygen atom from another water molecule. This is the very reason that water remains a liquid at Room Temperature (R.T) whereas its counterparts such as $\mathrm{H}_{2} \mathrm{~S}$, etc. are gases. The hydrogen bonding in water, together with its tendency to form open tetrahedral networks at low temperatures, gives rise to its characteristic properties, which differ from those of other liquids. Such properties of other liquids are often described as 'anamalous' although it could well be argued that water possesses properties that one might deduce from its structure. Quite small percentage changes in the strength of the aqueous hydrogen bond may give rise to large percentage changes in such physical properties as melting point, boiling point, density and viscosity. There is considerable hydrogen bonding in liquid water resulting in high cohesion which prevents water molecules from being easily released from the water 
surface. Consequentially, the vapor pressure is reduced and water has a high boiling point. The percentage reductions in the hydrogen bond strength that result in lower boiling points is given in graph below

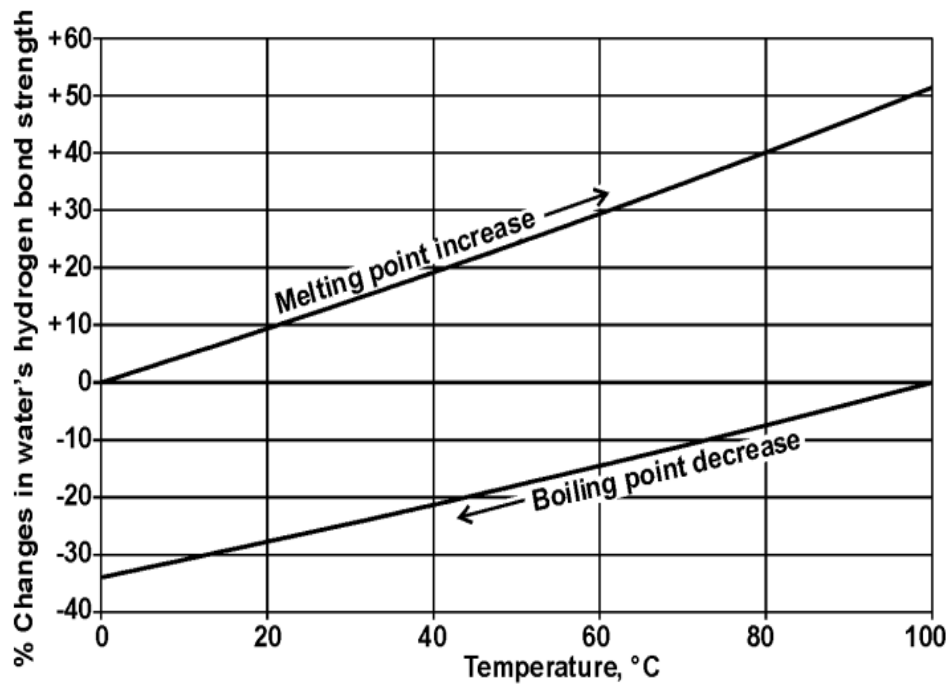

\section{FIGURE 1: CHANGE IN BOILING POINT OF WATER WITH \% REDUCTION IN H BONDING}

If the strength of this H-bonding is reduced even by a minor percentage then desalination can be performed at a temperature slightly over R.T. $\left(37^{\circ} \mathrm{C}\right)[5]$. Thus at a temperature little over R.T water can be completely separated from salts. The main advantage of using such a process would be that many sources are available for provision of energy to heat feed water to $37^{\circ}$ $\mathrm{C}$ thus reducing the costs incurred due to energy requirements to a bare minimum. Currently, uses of chaotropic agents such as Guanidium chloride, Sodium dodecyl sulfate, urea etc. are being investigated for this purpose.

TABLE 1

ESTIMATES OF EFFECTS CONSEQUENT ON VARYING WATER'S HYDROGEN BOND STRENGTH

\begin{tabular}{|c|c|}
\hline \% Change in hydrogen Bond & Effect at $37^{\circ} \mathbf{C}$ \\
\hline Decrease $29 \%$ & Water boils \\
\hline Decrease $18 \%$ & Most proteins heat denature \\
\hline Decrease $11 \%$ & $\mathrm{~K}+$ becomes kosmotropic \\
\hline Decrease $7 \%$ & pKw up 3...and so on \\
\hline
\end{tabular}

\section{CONCLUSION}

Desalination today is a major matter of concern, both for municipal supplies as well as the industry, especially in regions such as MENA where freshwater reserves are scarce. The demand for freshwater is predicted to increase exponentially in the coming decade thus also raising demands for alternative energy resources. Developing countries, currently having abundant water resources, are going to face scarcity in the near future due to over exploitation of this valuable natural resource. Also those which do not have access (geographical and economical) to sufficient energy resources are going to suffer if this trend continues [7].

Although current techniques used for desalination are being continuously optimized and economized, the tremendous energy requirements remain a major issue that needs to be catered. Novel technological innovations in ED by various technology providers are slowly giving it better acceptance over RO or other membrane technologies. Use of renewable sources to meet these requirements is a viable option but as of today none of those sources are extracted efficiently enough to meet the ever growing demands of this industry. In experiments conducted by us on a lab scale, the suggested chaotrops and kosmotrops have given us a mere drop of $5-10^{0} \mathrm{C}$. Hence this is currently not a full proof method for scaling up. The alternative suggested in this paper, if implemented on a large scale, can virtually eliminate the concern in relation with energy requirements. Further developments and finally implementation of this novel technique can be done in the near future thus solving the energy crisis that persists, at least in the field of desalination. 


\section{REFERENCES}

[1] Renewable energy desalination: An Emerging Solution to close the water gap in the Middle East and North Africa.

[2] Shahid, Chaudhry, An Overview of Industrial Desalination Technologies, ASME Industrial Demineralization (Desalination): Best Practices \& Future Directions Workshop, 2013; 1-30.

[3] Uchenna K. Kesieme, Nicholas Milne, Hal Aral, Chu Yong Cheng, Mikel Duke, Economic analysis of desalination technologies in the context of carbon pricing and opportunities for membrane distillation, Desalination, 2013; 323: 66-74.

[4] Akili D. Khawaji, Ibrahim K. Kutubkhanah, Jong-Mihn Wie, Advances in seawater desalination technologies, Desalination, 2008; 221: 47-69

[5] Martin Chaplin, Water's Hydrogen Bond Strength, Water's Hydrogen Bond Strength.

[6] Anurag Bajpayee, Tengfei Luo, Andrew Muto and Gang Chen, Very low temperature membrane free desalination by direct solvent extraction, Energy and Environmental Science, 2011; 4, 1672.

[7] Mohamed A. Eltawil, Zhao Zhengming, Liqiang Yuan, A review of renewable energy technologies integrated with desalination systems, Renewable and Sustainable Energy Reviews, 2009; 2245-2262. 